\title{
Optical properties of two-dimensional magnetoelectric point scattering lattices
}

\author{
Hansen, Per Lunnemann; Sersic, Ivana; Koenderink, A. Femius
}

Published in:

Physical Review B Condensed Matter

Link to article, DOI:

10.1103/PhysRevB.88.245109

Publication date:

2013

Document Version

Publisher's PDF, also known as Version of record

Link back to DTU Orbit

Citation (APA):

Hansen, P. L., Sersic, I., \& Koenderink, A. F. (2013). Optical properties of two-dimensional magnetoelectric point scattering lattices. Physical Review B Condensed Matter, 88(24), [245109].

https://doi.org/10.1103/PhysRevB.88.245109

\section{General rights}

Copyright and moral rights for the publications made accessible in the public portal are retained by the authors and/or other copyright owners and it is a condition of accessing publications that users recognise and abide by the legal requirements associated with these rights.

- Users may download and print one copy of any publication from the public portal for the purpose of private study or research.

- You may not further distribute the material or use it for any profit-making activity or commercial gain

- You may freely distribute the URL identifying the publication in the public portal

If you believe that this document breaches copyright please contact us providing details, and we will remove access to the work immediately and investigate your claim. 


\title{
Optical properties of two-dimensional magnetoelectric point scattering lattices
}

\author{
Per Lunnemann, ${ }^{1}$ Ivana Sersic, ${ }^{2}$ and A. Femius Koenderink ${ }^{2, *}$ \\ ${ }^{1}$ DTU Fotonik, Department of Photonics Engineering, Ørstedsplads 343, DK-2800, Denmark \\ ${ }^{2}$ Center for Nanophotonics, FOM Institute AMOLF, Science Park 104, 1098 XG Amsterdam, The Netherlands
}

(Received 26 September 2013; published 6 December 2013)

\begin{abstract}
We explore the electrodynamic coupling between a plane wave and an infinite two-dimensional periodic lattice of magnetoelectric point scatterers, deriving a semianalytical theory with consistent treatment of radiation damping, retardation, and energy conservation. We apply the theory to arrays of split ring resonators and provide a quantitative comparison of measured and calculated transmission spectra at normal incidence as a function of lattice density, showing excellent agreement. We further show angle-dependent transmission calculations for circularly polarized light and compare with the angle-dependent response of a single split ring resonator, revealing the importance of cross coupling between electric dipoles and magnetic dipoles for quantifying the pseudochiral response under oblique incidence of split ring lattices.
\end{abstract}

DOI: 10.1103/PhysRevB.88.245109

PACS number(s): 78.67.Pt, 78.20.Ls, 78.20.Fm

\section{INTRODUCTION}

Since the seminal works of Veselago ${ }^{1}$ and Pendry, ${ }^{2}$ much effort has been put into designing and fabricating artificial materials using periodic nanostructured materials with effective material parameters $\boldsymbol{\epsilon}$ and $\boldsymbol{\mu}$ that otherwise do not exist in nature. ${ }^{3}$ The mathematical tools of transformation optics ${ }^{4}$ state that full control over $\boldsymbol{\epsilon}$ and $\boldsymbol{\mu}$ allows nearly arbitrary rerouting of light through space, ${ }^{4}$ with exotic applications such as superlenses and cloaking. Besides tailoring of $\boldsymbol{\epsilon}$ and $\boldsymbol{\mu}$, the scattering properties of the subwavelength building blocks that were developed for metamaterials have attracted much attention. $^{5-13}$ Tailoring of the optical scattering properties may be achieved by structural design of the scatterers to control their electric and magnetic dipole polarizability, as well as by tuning their mutual optical coupling by changing their relative coordination and orientation. With recent advances in nanotechnological fabrication techniques, based on these principles, novel metasurfaces ${ }^{14,15}$ have been demonstrated, as well as compact and on-chip compatible optical antennas, ${ }^{16,17}$ waveguides, ${ }^{18}$ flat lenses, ${ }^{19,20}$ and materials with giant birefringence. $6,7,15,21-24$

Scattering experiments on metamaterials are frequently done on periodic planar arrays of scatterers with subdiffraction pitch. ${ }^{25-29}$ The chain of reasoning from measurement to effective media parameters generally starts from measured intensity reflection and transmission that are used to validate bruteforce finite difference time domain (FDTD) simulations. ${ }^{30-32}$ The FDTD calculations in turn lead to retrieval of effective parameters from the calculated amplitude reflection and transmission. ${ }^{27,28}$ In the vein of the classical Lorentz oscillator model, it is desirable to express an array response in terms of a polarizability per element, rather than in an effective $\boldsymbol{\epsilon}$ and $\boldsymbol{\mu}$. Indeed, it is now generally accepted that the split ring resonator (SRR), for instance, is a strongly polarizable electric and magnetic dipole scatterer, and that SRRs interact depending on their density, local lattice coordination, and relative orientation via near- and far-field dipole terms. ${ }^{5,6,8,11,27,29,33}$ Since split rings have extinction cross sections far in excess of the typical unit cell areas of the metamaterial lattices they are stacked in, and comparable to the unitary limit, ${ }^{29,34}$ coupling is not only via $1 / r^{3}$ near-field interactions, ${ }^{35}$ but also via retarded far-field terms. ${ }^{12,29,36}$ Indeed, transmission experiments on SRRs show strong superradiant broadening effects that increase with SRR density, ${ }^{8,29}$ and further depend on incidence angle. Decker et al. ${ }^{12}$ attempted to account for these interactions using numerical summation of retarded electric dipole-dipole interactions on a one-dimensional (1D) chain. However, in this approach, qualitative discrepancies remain compared to full numerical simulations, likely because numerical summation of dipole-dipole interactions in real space is poorly convergent ${ }^{37,38}$ because actual lattices in experiments are not $1 \mathrm{D}$ and because interactions also involve magnetic dipole-dipole coupling and magnetoelectric coupling. The minimum requirements for a simple dipole lattice model for metamaterials must necessarily include the electrodynamic coupling between electric dipoles, magnetic dipoles, as well as the cross coupling between magnetic and electric dipoles. Here, we propose a simple model that employs exponentially convergent dipole sums and can deal with infinite 2D periodic lattices, taking any physical magnetoelectric polarizability tensor as input. The benefit of such a model is that it predicts quantitative transmission and reflection spectra that can be directly matched to data studying diffraction effects, lattice resonances, or angle-dependent spatial dispersion effects. $^{5,8,11,12,27,29,33,39}$ Indeed our model is not limited to any specific range of periodicities or scattering strengths, encompassing both dense metamaterials as well as diffractive gratings.

This paper is organized as follows. In Sec. II, we generalize Ewald lattice sum techniques ${ }^{40}$ to point scatterers with a magnetoelectric $6 \times 6$ dynamic polarizability tensor, with interactions mediated by a $6 \times 6$ Green dyadic. ${ }^{36}$ In Sec. III A, we compare predicted normal-incidence transmission to measured spectra for square and rectangular SRR lattices. In Sec. III B, we present calculations for circular polarization at oblique incidence to evidence how single-building-block pseudochirality carries over into transmission asymmetry. ${ }^{41}$

\section{LATTICE THEORY}

\section{A. Polarizability tensor}

We consider a 2D lattice consisting of arbitrary magnetoelectric point scatterers each described by a polarizability 
tensor. By definition, the polarizability relates the induced electric and magnetic dipole moment, $\boldsymbol{p}$ and $\boldsymbol{m}$, in response to an electric and magnetic field $\boldsymbol{E}$ and $\boldsymbol{H}$ according to ${ }^{36}$

$$
\left(\begin{array}{c}
\boldsymbol{p} \\
\boldsymbol{m}
\end{array}\right)=\overleftrightarrow{\alpha}\left(\begin{array}{c}
\boldsymbol{E} \\
\boldsymbol{H}
\end{array}\right)
$$

The magnetoelectric polarizability may be conveniently written as

$$
\overleftrightarrow{\alpha}=\left(\begin{array}{ll}
\overleftrightarrow{\alpha}_{E E} & \overleftrightarrow{\alpha}_{E H} \\
\overleftrightarrow{\alpha}_{H E} & \overleftrightarrow{\alpha}_{H H}
\end{array}\right)
$$

where $\overleftrightarrow{\alpha}_{E E}$ is the $3 \times 3$ electric polarizability tensor that quantifies the induced electric dipole moment in response to an electric field. Similarly, $\stackrel{\leftrightarrow}{\alpha}_{H H}$ describes the magnetic polarizability that quantifies the induced magnetic dipole in response to a magnetic driving field. Finally, $\stackrel{\leftrightarrow}{\alpha}_{E H}$ denotes the magnetoelectric coupling that describes the induced electric dipole moment in response to a magnetic field, and vice versa. We shall denote $\stackrel{\leftrightarrow}{\alpha}$ the bare polarizability, since it describes the induced dipole moments in the absence of neighboring point scatterers. As treated in Ref. $36, \overleftrightarrow{\alpha}$ is subject to several constraints that we, for completeness, shall briefly summarize: Due to reciprocity, the polarizability is subject to the Onsager constraints ${ }^{42,43}$

$$
\overleftrightarrow{\alpha}_{E E}=\overleftrightarrow{\alpha}_{E E}^{\top}, \quad \overleftrightarrow{\alpha}_{H H}=\overleftrightarrow{\alpha}_{H H}^{\top}, \quad \overleftrightarrow{\alpha}_{E H}=-\overleftrightarrow{\alpha}_{H E}^{\top}
$$

where the superscripted $T$ denotes matrix transpose. Moreover, energy conservation constrains the dynamic polarizability, in case of no Ohmic loss, to fulfill an optical theorem of the Sipe-Kranendonk ${ }^{44}$ form

$$
\frac{1}{2 i}\left[\overleftrightarrow{\alpha}-\overleftrightarrow{\alpha}^{* \top}\right]=\frac{2}{3} k^{3} \overleftrightarrow{\alpha}^{* \top} \overleftrightarrow{\alpha},
$$

as derived by Belov et al., ${ }^{45}$ and later by Sersic et al. ${ }^{36} \mathrm{~A}$ different way of writing this constraint is that the scalar optical theorem $\operatorname{Im} \alpha \leqslant 2 / 3 k^{3}|\alpha|^{2}$ must hold for each eigenvalue of $\overleftrightarrow{\alpha}$, where equality holds in the absence of loss. ${ }^{36}$ Any electrostatic bare polarizability tensor $\overleftrightarrow{\alpha}_{0}$, such as that derived from an Ohmically damped $L C$-circuit model, may be turned into a bona fide electrodynamic polarizability that is bound by the optical theorem in Eq. (4) by addition of radiation damping,

$$
\overleftrightarrow{\alpha}^{-1}=\overleftrightarrow{\alpha}_{0}^{-1}-\frac{2}{3} k^{3} i \mathbb{I}
$$

where $k$ denotes the wave vector, $\mathbb{I}$ is the six-dimensional identity tensor, and $(\cdot)^{-1}$ denotes matrix inversion.

\section{B. Lattice response}

We consider the response to plane-wave illumination of a 2D periodic lattice of point scatterers, which is defined by a set of lattice vectors $\boldsymbol{R}_{m n}=m \boldsymbol{a}_{1}+n \boldsymbol{a}_{2}$ (where $m$ and $n$ are integers and $\boldsymbol{a}_{1,2}$ are the real-space basis vectors; see Fig. 1). The response of a particle at position $\boldsymbol{R}_{m n}$ is self-consistently set by the incident field, plus the field of all other dipoles in

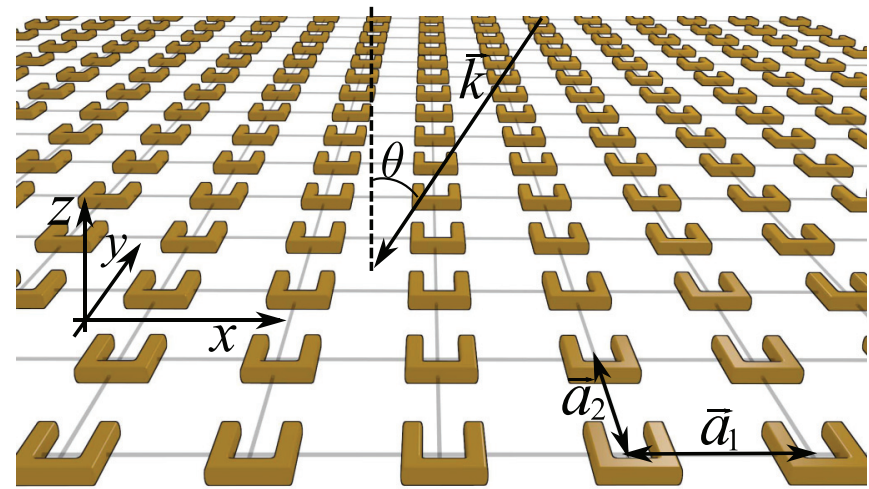

FIG. 1. (Color online) Illustration of the considered lattice, here sketched for split ring resonators, with a plane-wave incident at an angle $\theta$.

the lattice according to ${ }^{40}$

$$
\begin{aligned}
\left(\begin{array}{c}
\boldsymbol{p}_{m n} \\
\boldsymbol{m}_{m n}
\end{array}\right)= & \overleftrightarrow{\alpha}\left[\left(\begin{array}{c}
\boldsymbol{E}_{\mathrm{in}}\left(\boldsymbol{R}_{m n}\right) \\
\boldsymbol{H}_{\mathrm{in}}\left(\boldsymbol{R}_{m n}\right)
\end{array}\right)+\sum_{m^{\prime} \neq m, n^{\prime} \neq n} \overleftrightarrow{G}^{0}\left(\boldsymbol{R}_{m n}-\boldsymbol{R}_{m^{\prime} n^{\prime}}\right)\right. \\
& \left.\times\left(\begin{array}{c}
\boldsymbol{p}_{m^{\prime} n^{\prime}} \\
\boldsymbol{m}_{m^{\prime} n^{\prime}}
\end{array}\right)\right]
\end{aligned}
$$

where $\overleftrightarrow{G}^{0}\left(\boldsymbol{R}_{m n}-\boldsymbol{R}_{m^{\prime} n^{\prime}}\right)$ is the $6 \times 6$ dyadic Green function of the medium surrounding the split ring lattice. In this work, we take the surrounding medium to be homogeneous space.

For plane-wave incidence with wave vector $\boldsymbol{k}_{\|}$and using translation invariance of the lattice, we can substitute a Bloch wave form $\left(\boldsymbol{p}_{m n}, \boldsymbol{m}_{m n}\right)^{T}=e^{i \boldsymbol{k}_{\|} \cdot \boldsymbol{R}_{m n}}\left(\boldsymbol{p}_{00}, \boldsymbol{m}_{00}\right)^{T}$ to obtain

$$
\left(\begin{array}{c}
\boldsymbol{p}_{00} \\
\boldsymbol{m}_{00}
\end{array}\right)=\left[\overleftrightarrow{\alpha}^{-1}-\overleftrightarrow{\mathcal{G}}^{\neq}\left(\boldsymbol{k}_{\|}, 0\right)\right]^{-1}\left(\begin{array}{c}
\boldsymbol{E}_{\mathrm{in}}\left(\boldsymbol{R}_{00}\right) \\
\boldsymbol{H}_{\mathrm{in}}\left(\boldsymbol{R}_{00}\right)
\end{array}\right) \text {. }
$$

Here, $\overleftrightarrow{\mathcal{G}}^{\neq}\left(\boldsymbol{k}_{\|}, 0\right)$ is a summation of the dyadic Green function $\stackrel{\leftrightarrow}{G}^{0}$ over all positions on the 2D periodic real-space lattice barring the origin:

$$
\stackrel{\leftrightarrow}{\mathcal{G}}^{\neq}\left(\boldsymbol{k}_{\|}, \boldsymbol{r}\right)=\sum_{m \neq 0, n \neq 0} \stackrel{\leftrightarrow}{G}^{0}\left(\boldsymbol{R}_{m n}-\boldsymbol{r}\right) e^{i \boldsymbol{k}_{\|} \cdot \boldsymbol{R}_{m n}} .
$$

We will refer to the summation without exclusion of $m=n=0$ as $\overleftrightarrow{\mathcal{G}}\left(\boldsymbol{k}_{\|}, \boldsymbol{r}\right)$. We immediately identify the factor $\left[\overleftrightarrow{\alpha}^{-1}-\overleftrightarrow{\mathcal{G}}^{\neq}\left(\boldsymbol{k}_{\|}, 0\right)\right]^{-1}$ to be an effective polarizability tensor of the SRR, renormalized by the lattice interactions. This is equivalent to the formulation that is didactically explained by García de Abajo in Ref. 40, however, now generalized to the magnetoelectric case. Importantly, the summed lattice interactions not only renormalize the magnitude of $\alpha$, but also the relative strength of the electric and magnetic terms, and the magnetoelectric cross coupling. Since we are not aware of any reported implementation of lattice sums for the $6 \times 6$ dyadic Green function $\stackrel{\leftrightarrow}{G}^{0}$, we supply full details in Appendix A. The challenging nature of the summations lies in the fact that dipole sums are poorly convergent as a real-space summation due to the fact that the Green function only has a $1 / r$ dropoff. To overcome this, we directly follow the formulation by Linton, ${ }^{38}$ splitting the summation into a real-space part and a reciprocal-space part that both converge exponentially. While 
the work by Linton treats the Green function of the scalar Helmholtz equation, ${ }^{38}$ the necessary steps for expanding it to the $6 \times 6$ dyadic Green functions are easily derived.

\section{Far field}

Once one has obtained the induced dipole moments, given the incident field, the field distribution immediately follows as ${ }^{40}$

$$
\left(\begin{array}{c}
\boldsymbol{E}(\boldsymbol{r}) \\
\boldsymbol{H}(\boldsymbol{r})
\end{array}\right)=\left(\begin{array}{c}
\boldsymbol{E}_{\mathrm{in}} \\
\boldsymbol{H}_{\mathrm{in}}
\end{array}\right) e^{i \boldsymbol{k} \cdot \boldsymbol{r}}+\mathcal{G}\left(\boldsymbol{k}_{\|}, \boldsymbol{r}\right)\left(\begin{array}{c}
\boldsymbol{p}_{00} \\
\boldsymbol{m}_{00}
\end{array}\right),
$$

where the second term describes the scattered field. To find the reflected and transmitted far-field amplitudes, we note that for an observation point in the far field, the Green function can be written as ${ }^{35}$

$$
\overleftrightarrow{G}^{0}\left(\boldsymbol{r}-\boldsymbol{R}_{m n}\right)=k^{2} \frac{\exp \left(i k\left|\boldsymbol{r}-\boldsymbol{R}_{m n}\right|\right)}{\left|\boldsymbol{r}-\boldsymbol{R}_{m n}\right|} \overleftrightarrow{M}_{m n}^{(\infty)}
$$

where $\overleftrightarrow{M}_{m n}^{(\infty)}$ is a dimensionless matrix with elements of order unity that only depends on the direction and not the length of $\boldsymbol{r}-\boldsymbol{R}_{m n}$, and which we list in Appendix B. Taking the scattered field as the sum over all lattice points,

$$
\left(\begin{array}{c}
\boldsymbol{E}_{s}(\boldsymbol{r}) \\
\boldsymbol{H}_{s}(\boldsymbol{r})
\end{array}\right)=\sum_{n, m} k^{2} \frac{\exp \left(i k\left|\boldsymbol{r}-\boldsymbol{R}_{n m}\right|\right)}{\left|\boldsymbol{r}-\boldsymbol{R}_{n m}\right|} e^{i \boldsymbol{k}_{||} \cdot \boldsymbol{R}_{n m}} \overleftrightarrow{M}_{n m}^{(\infty)}\left(\begin{array}{c}
\boldsymbol{p}_{00} \\
\boldsymbol{m}_{00}
\end{array}\right),
$$

we make the far-field expansion, assuming that the orientational factor $\overleftrightarrow{M}_{m n}^{(\infty)}$ does not vary with $(n, m)$ and using the identity

$$
\frac{\exp \left(i k\left|\boldsymbol{r}-\boldsymbol{R}_{m n}\right|\right)}{\left|\boldsymbol{r}-\boldsymbol{R}_{m n}\right|}=\frac{i}{2 \pi} \int d \boldsymbol{q} \frac{\exp \left[i \boldsymbol{q} \cdot\left(\boldsymbol{r}_{\|}-\boldsymbol{R}_{m n}\right)+k_{z} z\right]}{k_{z}},
$$

with $k_{z}=\sqrt{k^{2}-|\boldsymbol{q}|^{2}}$. Furthermore, we use the completeness relation of the lattice

$$
\sum_{m, n} e^{i \boldsymbol{k}_{\|} \cdot \boldsymbol{R}_{m n}}=\frac{(2 \pi)^{2}}{\mathcal{A}} \sum_{\tilde{m}, \tilde{n}} \delta\left(\boldsymbol{k}_{\|}-\boldsymbol{g}_{\tilde{m} \tilde{n}}\right),
$$

where $\mathcal{A}$ is the real-space unit cell surface area spanned by the basis vectors $\boldsymbol{a}_{1}$ and $\boldsymbol{a}_{2}$ and $\boldsymbol{g}_{\tilde{m} \tilde{n}}=\tilde{m} \boldsymbol{b}_{1}+\tilde{n} \boldsymbol{b}_{2}$, with $\boldsymbol{b}_{1,2}$ being the reciprocal lattice basis vectors. Inserting Eqs. (12) and (13) into (11), one retrieves diffracted orders in the far field of the form

$$
\left(\begin{array}{l}
\boldsymbol{E}_{s}(\boldsymbol{r}) \\
\boldsymbol{H}_{s}(\boldsymbol{r})
\end{array}\right)=\sum_{\tilde{m} \tilde{n},\left|\boldsymbol{k}_{\tilde{m} \tilde{n}}\right| \leqslant k}\left(\begin{array}{c}
\boldsymbol{E}_{\tilde{m} \tilde{n}} \\
\boldsymbol{H}_{\tilde{m} \tilde{n}}
\end{array}\right) e^{i \boldsymbol{k}_{\tilde{m} \tilde{n}} \cdot \boldsymbol{r}},
$$

where $\boldsymbol{k}_{\tilde{m} \tilde{n}}=\left(\boldsymbol{k}_{\|}+\boldsymbol{g}_{\tilde{m} \tilde{n}}, \pm \sqrt{k^{2}-\left|\boldsymbol{k}_{\|}+\boldsymbol{g}_{\tilde{m} \tilde{n}}\right|^{2}}\right)=k\left(\cos \phi_{\tilde{m} \tilde{n}}\right.$ $\left.\sin \theta_{\tilde{m} \tilde{n}}, \sin \phi_{\tilde{m} \tilde{n}} \sin \theta_{\tilde{m} \tilde{n}}, \cos \theta_{\tilde{m} \tilde{n}}\right)$ are the diffracted wave vectors. The fields associated with each order are

$$
\left(\begin{array}{c}
\boldsymbol{E}_{\tilde{m} \tilde{n}} \\
\boldsymbol{H}_{\tilde{m} \tilde{n}}
\end{array}\right)=\frac{i 2 \pi k}{A \cos \theta_{\tilde{m} \tilde{n}}} \overleftrightarrow{M}\left(\theta_{\tilde{m} \tilde{n}}, \phi_{\tilde{m} \tilde{n}}\right)\left(\begin{array}{c}
\boldsymbol{p}_{00} \\
\boldsymbol{m}_{00}
\end{array}\right) \text {. }
$$

Using Eq. (14), for a field incident with angles $(\theta, \phi)$, we may calculate the transmitted far-field intensity as $I_{t}=$ $-\frac{1}{2 Z_{0}} \operatorname{Re}[\boldsymbol{E}(\theta, \phi) \times \boldsymbol{H}(\theta, \phi)] \cdot \hat{z}$, where $(\boldsymbol{E}, \boldsymbol{H})$ denotes the sum of the incoming $\left(\boldsymbol{E}_{\text {in }}, \boldsymbol{H}_{\text {in }}\right)$ and forward-scattered field $\left(\boldsymbol{E}_{s}, \boldsymbol{H}_{s}\right)$ and $Z_{0}=\sqrt{\epsilon / \mu}$ is the impedance of the background material. Similarly, the reflected field is found as $I_{r}=\frac{1}{2 Z_{0}} \operatorname{Re}\left[\boldsymbol{E}_{s}(\pi-\right.$ $\left.\theta, \phi) \times \boldsymbol{H}_{s}(\pi-\theta, \phi)\right] \cdot \hat{z}$. Dividing the incident intensity with the reflected/transmitted intensity, we obtain the intensity reflection/transmission coefficients. For sufficiently large pitch, grating diffraction orders will appear. For the common case of planar magnetic scatterers such as split rings, where the magnetic dipole moment must be perpendicular to the 2D plane and the electric dipole must be along $x$, the normal-incidence zero-order amplitude transmission reduces to

$$
t_{x x}=1+\frac{2 \pi i k}{\mathcal{A}}\left[\frac{1}{1 / \overleftrightarrow{\alpha}-\overleftrightarrow{\mathcal{G}}^{\neq}\left(\boldsymbol{k}_{\|}=0,0\right)}\right]_{11},
$$

where the subscripted 11 denotes the first row and first column entry of the matrix, and the subscript $x x$ indicates transmission in the $x$-polarized output channel given $x$-polarized input light.

\section{RESULTS}

\section{A. Linear polarization}

To verify how far the simple model, presented in Sec. II, captures the transmission properties of actual metamaterials, we compare calculated transmission spectra to measurements reported in Ref. 29 on $\mathrm{Au}$ split ring resonator lattices with dimensions $200 \times 200 \times 30 \mathrm{~nm}$. These split rings (made using electron-beam lithography and lift-off) have a split width of $80 \mathrm{~nm}$ between the two arms. With the geometry illustrated in Fig. 1, we use a polarizability tensor of the form

$$
\overleftrightarrow{\alpha}_{0}=\mathcal{L}(\omega)\left(\begin{array}{ccccc}
\eta_{E} & 0 & \ldots & 0 & i \eta_{C} \\
0 & 0 & & & 0 \\
\vdots & & \ddots & & \vdots \\
0 & & & 0 & 0 \\
-i \eta_{C} & 0 & \ldots & 0 & \eta_{H}
\end{array}\right),
$$

where $\mathcal{L}(\omega)$ is a Lorentzian prefactor,

$$
\mathcal{L}(\omega)=V \frac{\omega_{0}^{2}}{\omega_{0}^{2}-\omega^{2}-i \omega \gamma},
$$

where $\gamma$ describes the damping rate due to Ohmic losses and $V$ is the physical SRR volume. Within the chosen unit system, ${ }^{36}$ the quantities $\eta_{E}, \eta_{H}$, and $\eta_{C}$ are dimensionless and directly comparable in magnitude. We emphasize that Eq. (16) describes the polarizability of a resonance from a single SRR that can be extracted from single SRR scattering experiments. The value of $\eta_{E}$ has been extracted earlier from a single $\mathrm{SRR},{ }^{34}$ but retrieving all tensor elements requires angle resolved scattering experiments of a single SRR as described in Ref. 36. Furthermore, the values have been retrieved from a full wave simulation; ${ }^{46}$ however, in this case, the glass interface was not included in the model. Finally, for implementation, we note that the polarizability tensor in Eq. (16) is not strictly invertible. This problem may be amended either by limiting the calculation to the $\left(E_{x}, H_{z}\right)$ subspace, by employing the Moore-Penrose pseudoinverse, or by substituting a small polarizability for the zeros on the diagonal. We use the latter method. Higher-order resonances can be added to the electrostatic polarizability prior to applying the radiation damping correction.

Figure 2(a) shows measured normal-incidence transmission versus wavelength for square lattices with pitches ranging 


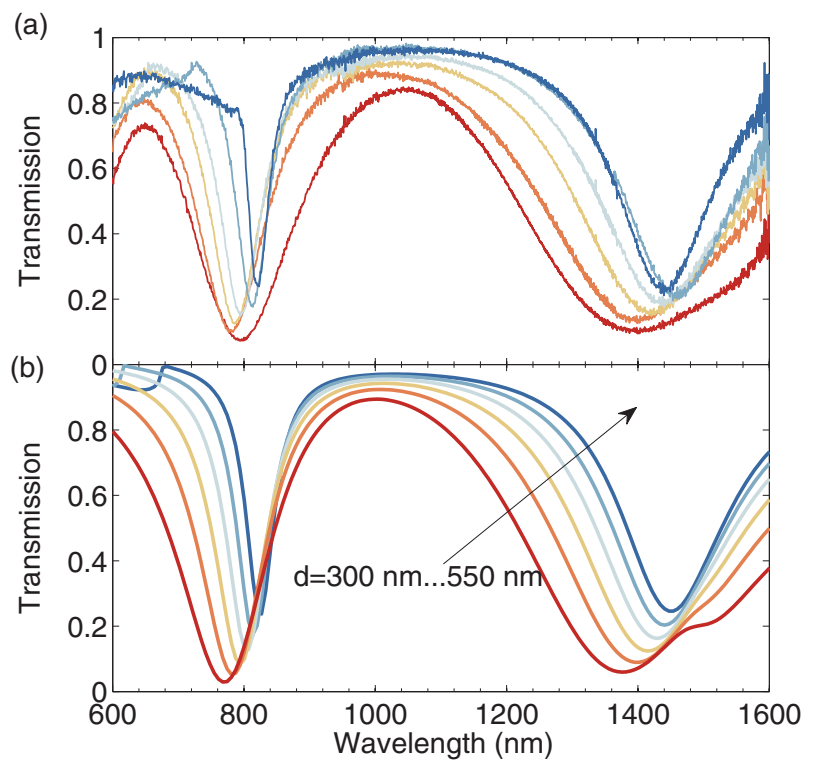

FIG. 2. (Color online) (a) Measured transmission spectrum for a normal-incidence field linearly polarized along $x$ lattice spacings between 300 to $550 \mathrm{~nm}$ in steps of $50 \mathrm{~nm}$. (see Fig. 1 for geometry sketch). (b) Corresponding fitted transmission spectrum using $\left(\eta_{E}, \eta_{H}, \eta_{C}\right)=(0.40,0.26,0.33)$ and $\lambda_{0}=1.52 \mu \mathrm{m}$ for the magnetic resonance and $\left(\eta_{E}, \eta_{H}, \eta_{C}\right)=(0.17,0.00,0.00)$ and $\lambda_{1}=0.82 \mu \mathrm{m}$ for the plasmonic resonance using $V=200 \times 200 \times 30 \mathrm{~nm}$ and $\gamma=8.3 \times 10^{13} \mathrm{~s}^{-1}$.

from 300 to $550 \mathrm{~nm}$, while Fig. 2(b) shows the corresponding calculated spectra. The data is reproduced from Ref. 29. Two distinct resonances are observed near 1500 and $800 \mathrm{~nm}$ that are associated with, respectively, the $L C$ magnetic resonance and a higher-order plasmonic resonance, respectively. ${ }^{28,33}$ For the most dilute lattices, the higher-order resonance overlaps with a Rayleigh anomaly, i.e., the emergence of a grating diffraction order into the glass substrate. Based on the lattice sum theory, presented in Sec. II, we calculated the transmission spectrum for comparison, taking the static polarizability as the sum $\overleftrightarrow{\alpha}_{a}\left(\omega_{a}\right)+\overleftrightarrow{\alpha}_{b}\left(\omega_{b}\right)$ of two resonances [Eq. (16)]. For both resonances, we use a set of parameters, $\eta_{E}, \eta_{H}, \eta_{C}$, and $\omega_{0}$ common to all lattice spacings, which we obtain by fitting all six measured spectra simultaneously by minimizing the sum of squared residuals over the entire measured wavelength range. In Fig. 2(b), the corresponding calculated transmission spectra are presented using fitted parameters $\left(\eta_{E}, \eta_{H}, \eta_{C}\right)=(0.40,0.26,0.33)$ and $\lambda_{0}=1.52 \mu \mathrm{m}$ for the magnetic resonance and $\left(\eta_{E}, \eta_{H}, \eta_{C}\right)=(0.17,0.00,0.00)$ and $\lambda_{1}=0.82 \mu \mathrm{m}$ for the plasmonic resonance. We discuss the confidence in these parameters further below. Throughout this entire paper, the damping rate of gold, SRR volume, and refractive index of the surrounding medium were not fitted but fixed at $\gamma=8.3 \times 10^{13} \mathrm{~s}^{-1}, V=200 \times 200 \times 30 \mathrm{~nm}$, and $n=1.23$. The value $n=1.23$ reflects the average refractive index between glass and air, and is used because the lattice sum formulation as reported here cannot include the actual asymmetric environment, i.e., the air-glass interface on which the split rings are situated.

From Fig. 2, we notice that the lattice sum model reproduces all features observed in the experimental data. Focusing on the magnetic resonance, it clearly predicts the broadening and blueshift of the resonance for decreasing pitch. From the calculated transmission, we observe a second shoulder emerging for the largest density, which is only barely resolved in the experimental data. Such a resonance splitting is expected since the single SRR resonance is associated with two frequency-degenerate eigenpolarizabilities, each being a different coherent superposition of $\boldsymbol{p}$ and $\boldsymbol{m} \cdot{ }^{36}$ Increasing the density increases the magnetoelectric dipole-dipole coupling between SRRs, which lifts the degeneracy. In terms of the dynamic on-resonance polarizabilities, the fitted parameters translate into $\left|\alpha_{E}\right|=3.8 \mathrm{~V},\left|\alpha_{H}\right|=2.5 \mathrm{~V}$, and $\left|\alpha_{C}\right|=3.2 \mathrm{~V}$. The extracted parameters indicate that the $L C$ resonance is primarily electric in nature, and that the bianisotropy $\eta_{C}$ makes it significantly easier for the electric field to induce a magnetic dipole than it is for the magnetic field.

To quantify the agreement between our calculation methods and previously reported measurement data, we extracted the center frequency, the resonance linewidth, and the extinction cross section of the magnetic resonance on three types of sample sets: one with a square grid where both $d_{x}$ and $d_{y}$ were changed equally over each sample, one with a rectangular grid with $d_{x}=500 \mathrm{~nm}$ and $d_{y}$ varying, and similarly one with a rectangular grid where $d_{y}=500 \mathrm{~nm}$ and $d_{x}$ varying. In order to correct for the well-known electron-beam lithography artifact that object density affects the required dose for realizing a specific feature, i.e., the so-called proximity effect, we fabricated samples at different electron-beam dose factors and used image analysis software to select arrays in which SRRs had identical dimensions (arm length, base length, gap width, gap depth) to within better than $5 \mathrm{~nm}$. As reported in Ref. 29, the gap between the arms for this set of samples is significantly larger $(100 \mathrm{~nm})$ than it is for ones presented in Fig. $2(80 \mathrm{~nm})$. The center frequency, resonance linewidth, and effective extinction cross section were extracted from the data by fitting a Lorentzian to the transmission resonance. The effective extinction cross section per split ring is defined as

$$
\sigma_{\mathrm{ext}}=\left(1-T_{\min }\right) d_{x} d_{y} \cos \theta,
$$

with $T_{\min }$ being the measured value of transmission at the transmission minimum, and $\theta$ being the angle of incidence that for this section is set to $0^{\circ}$. To evaluate the theory, we follow a procedure identical to the one followed for Fig. 2. In particular, the parameters $\eta_{E}, \eta_{H}, \eta_{C}$, and $\omega_{0}$ were obtained by simultaneously fitting all measured spectra of both square and rectangular lattices, by minimizing the sum of the summed squared residual of each transmission spectrum. Subsequent to fitting the spectra, the center frequency, resonance linewidth, and extinction cross section were extracted from the calculated transmission spectra exactly as done for the experimental measurements. Figure 3 shows the density dependence of the transmission resonance frequency, linewidth, and effective SRR extinction cross section, respectively, as predicted by the lattice sum model, together with the values extracted from experiment. The lattice sum calculation qualitatively reproduces the blueshift (redshift) when varying $d_{x}\left(d_{y}\right)$. As discussed in Ref. 29, the SRR consist of both a magnetic dipole pointing along $\hat{z}$ and an electric dipole along $\hat{\boldsymbol{x}}$. Aligned dipoles perpendicular to the lattice, i.e., the magnetic dipoles, will give rise to a blueshift upon increasing density as the 


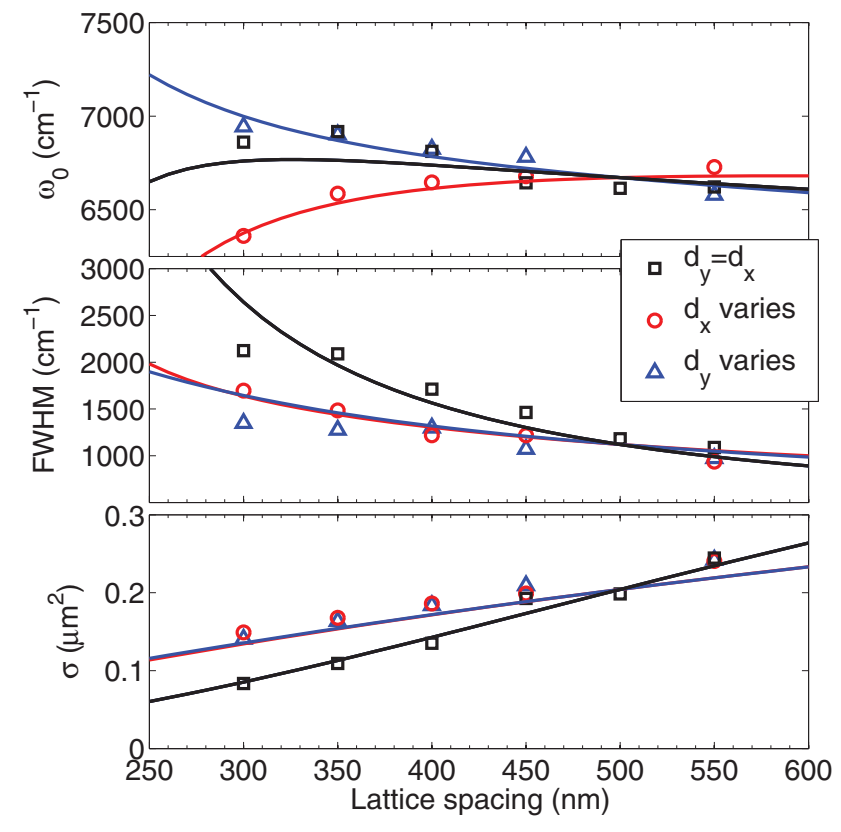

FIG. 3. (Color online) Comparison of calculated (lines) and measured (markers) (a) center frequency, (b) resonance linewidth, and (c) extinction cross section per split ring. Corresponding fit parameters were $\left(\eta_{E}, \eta_{H}, \eta_{C}\right)=(0.63 \pm 0.01,0.12 \pm 0.02,0.28 \pm 0.03)$ and $\lambda_{0}=1.57 \pm 0.003 \mu \mathrm{m}$ setting $V=200 \times 200 \times 30 \mathrm{~nm}$ and $\gamma=$ $8.3 \times 10^{13} \mathrm{~s}^{-1}$. In all plots, the line color and symbol shape indicate square lattices (square symbol, black lines), lattices with $d_{x}$ fixed to $500 \mathrm{~nm}$ and $d_{y}$ varying (red circular symbol), and vice versa (blue triangular symbol).

coupling is transverse. This shift has to compete with the resonance shift due to coupling between electric dipoles that has both a longitudinal (redshifting upon increasing density) contribution (head to tail coupling along $\hat{\boldsymbol{x}}$ ) and a transverse blueshifting (coupling along $\hat{\boldsymbol{y}}$ ) one. For lattices with large $d_{x}$ (fixed) and decreasing $d_{y}$, the mode simply blueshifts as both the electric and magnetic coupling are transverse. For lattices with large $d_{y}$ and decreasing $d_{x}$, the mode shifts to the red, as the longitudinal electric-dipole coupling is stronger than the transverse magnetic coupling. Finally, for the case of a square array $d_{x}=d_{y}$, the electric and magnetic transverse coupling is partially canceled by the electric longitudinal coupling, leading to a reduced blueshift. We further note that for the square lattice, we observe some discrepancy for the shortest lattice constants. We attribute this discrepancy to the fact that the shortest pitch square lattice sample is the densest, with spacing between split rings approximately half their diameter. From estimates for coupled plasmon particles, at and below this spacing, the dipole approximation breaks down ${ }^{47}$ Considering the resonance linewidth in Fig. 3(b), we first note that since the Ohmic damping does not depend on the coupling in an electrostatic model, the FWHM broadening with decreasing lattice spacing can only be explained by the radiation damping in an electrodynamic picture, which the lattice sum model fully takes into account and is in excellent agreement with the measurements thereof. Finally, the trend of a marked increase of effective cross section with reduced density is evident, with excellent agreement between theory and measurement. The meaning of the strong dependence of the effective cross section per split ring on pitch is that the effective cross section is bounded from above by the single split ring extinction cross section $\left(\sim 0.3 \mu \mathrm{m}^{2}\right.$; see Ref. 34) for dilute lattices and by the unit cell area for dense lattices. As the lattice is made denser, the unit cell area becomes smaller than the single object cross section. As the unit cell area is further decreased, super-radiant damping sets in that increases the FWHM and at the same time diminishes the effective extinction per split ring to be essentially pinned at the unit cell area.

We note that the theoretical values of the center frequency, linewidth, and extinction cross section were extracted by fitting full spectra, i.e., by performing a nonlinear least-squares fit to match measured and calculated frequency-dependent transmission $T(\omega)$. An alternative fit procedure would be to not base the fit merit function on the deviation in $T(\omega)$, but rather to only fit center frequency, width, and extinction cross section as extracted from data to those extracted from calculated spectra. On the basis of the fit, we conclude that the parameters that best describe the experiment are $\left(\eta_{E}, \eta_{H}, \eta_{C}\right)=(0.63 \pm$ $0.01,0.12 \pm 0.02,0.28 \pm 0.03)$ and $\lambda_{0}=1.57 \pm 0.003 \mu \mathrm{m}$, where the stated accuracies are the $95 \%$ confidence interval. The parameters are somewhat different from those obtained from the experiment in Fig. 2, and correspond to on-resonance dynamic polarizabilities of $\left|\alpha_{E}\right|=4.5 \mathrm{~V},\left|\alpha_{H}\right|=0.82 \mathrm{~V}$, and $\left|\alpha_{C}\right|=2.0 \mathrm{~V}$. We attribute the larger ratio between electric and magnetic response to the larger split width. We found that relaxing the constraint in the fit to the requirement that only the three extracted parameters, and not necessarily the entire spectrum, be fitted optimally in the least-squares sense does not yield a substantially improved value for the $\eta$ parameters. Ultimately, the reliability of the parameters is limited by our treatment of the dielectric environment. While the environment is, in fact, asymmetric (air-glass interface), we take the environment to be homogeneous with the index equal to the average of both media.

\section{B. Circular polarization}

As discussed in Ref. 36, the single SRR eigenpolarizabilities and eigenvectors of the polarizability tensor have special significance. In particular, the eigenpolarizabilities point to a largest and a smallest extinction cross section that can be addressed if the illumination field is chosen to equal the correct coherent mixture of $E_{x}$ and $H_{z}$ that the eigenvector prescribes. When $\left|\eta_{C}\right|>0$, the eigenvectors of the polarizability tensor correspond to oblique incidence circularly polarized light, implying a handed response in scattering. The existence of "bianisotropy," i.e., $\eta_{C} \neq 0$, was known from the outset in the field of metamaterials. ${ }^{48}$ As predicted in Ref. 36, and realized experimentally in Ref. 41, the strength of this effect can be directly probed using circular polarized light. Here we present lattice sum transmission calculations using circular polarized incident light that confirm a strongly handed extinction under oblique incidence. ${ }^{41}$ This comparison has no adjustable parameter since we take as input the polarizability tensor retrieved from the normal-incidence density-dependent data discussed above.

Figure 4 shows the calculated transmission spectrum for various incident angles using the same parameters as for Fig. 2. First, we note that the transmission spectra, when changing 


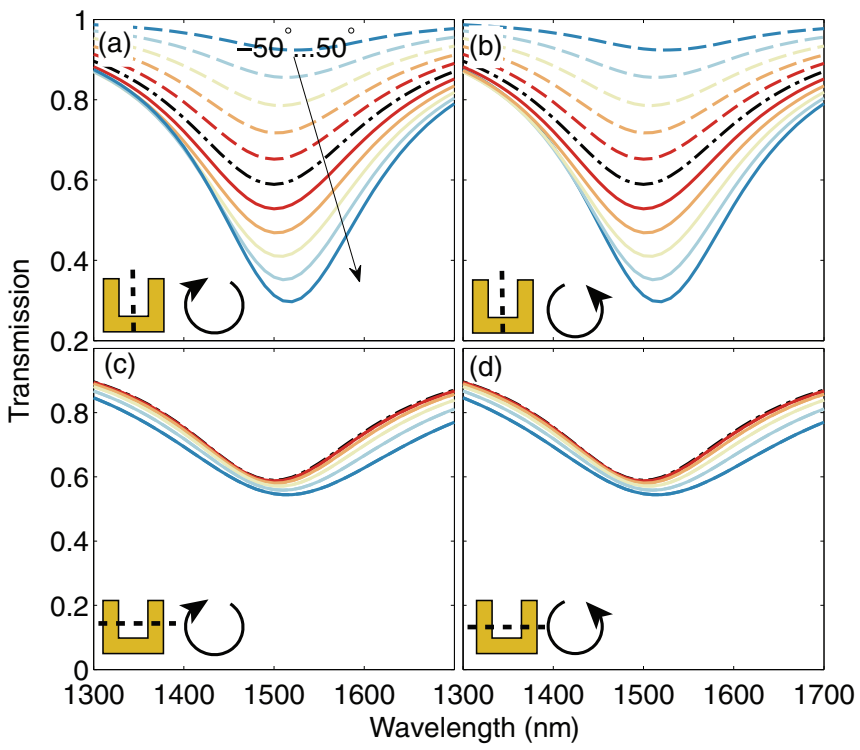

FIG. 4. (Color online) Calculated transmission vs wavelength for circularly polarized light incident at angles from $-50^{\circ}$ to $50^{\circ}$ in steps of $10^{\circ}$ for a square lattice with $d=500 \mathrm{~nm}$. Negative (positive) angles are plotted as dashed (solid) lines and normal incidence is marked as dash-dotted black lines. (a),(b) Right- (left-)hand polarized light with incident angle with rotational axis along the symmetry axis of the SSR, as depicted in the inset. (c),(d) Right- (left-)hand polarized light with incident angle with rotational axis perpendicular to the symmetry axis of the SRR, as depicted in the inset.

the incident angles around the symmetry axis of the SRR [Figs. 4(a) and 4(b)], reveal a strong angular dependence, while incident angles perpendicular to the symmetry axis reveal only a weak angular dependence. The strong angular dependence is strongly asymmetric around normal incidence, with transmission going from barely suppressed to very strong extinction when going from negative to positive angles for right-handed polarization (reversed behavior for opposite handedness). This is consistent with experimental results in Ref. 41. From a $L C$-circuit point of view, at oblique incidence angles the split ring is driven both by $E_{x}$ and $H_{z}$, and the handedness determines whether the phase difference between the $E_{x}$ and the $H_{z}$ field is such that the two driving terms for the capacitor and the current loop add up, or cancel. For rotations perpendicular to the symmetry axis [Figs. 4(c) and 4(d)], no such phase difference is present. On the basis of group theory arguments, it was first noted by Verbiest et al. ${ }^{49}$ that indeed a two-dimensional lattice can show optical activity in spite of the building block being achiral. This was later referred to as an extrinsic optical activity by Plum et al. ${ }^{7}$ and pseudochirality by Tretyakov et al. ${ }^{50}$ and Sersic et al. ${ }^{41}$

It has been argued by Gompf et al..$^{39}$ that for lattices with $d \sim \lambda$ spatial dispersion, an effect that is fully contained in our lattice sum approach, may conspire to induce handedness in the transmission, regardless of the shape of the building block of the lattice. However, with the disappearance of optical activity for rotations perpendicular to the symmetry axis, seen in Figs. 4(c) and 4(d), we conclude that it is indeed the building block that causes the handed behavior. The fact that the contrast in transmission is large is due to the fact that one of the two

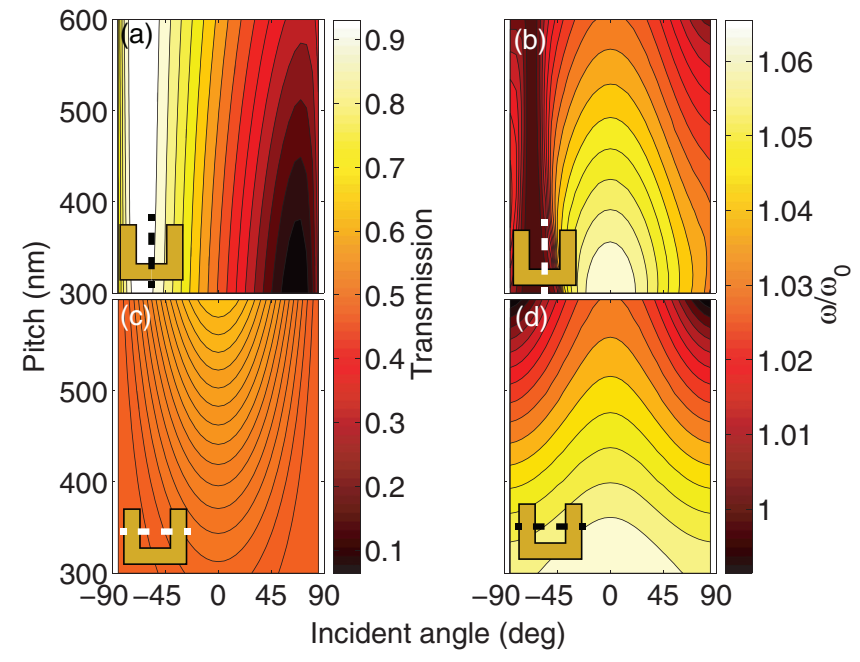

FIG. 5. (Color online) Left: Calculated minimum transmission, $T_{\min }$, of a square lattice as a function of incident angle and lattice pitch, for an incident right-hand polarized field. Right: The associated resonance frequency normalized with the single SRR resonance frequency. Top (bottom) row: incident angle with rotational axis parallel (perpendicular) to the symmetry axis of the SSR, as depicted in the inset.

eigenvalues of the split ring polarizabilities vanishes at the given large cross coupling.

In Figs. 5(a) and 5(b), the calculated minimum transmission, $T_{\min }$, is plotted as a function of incident angle and lattice pitch, for right-handed input polarization and incident angle with rotational axis parallel (perpendicular) to the symmetry axis of the SSR. Considering Fig. 5(a), we first note that for any given lattice spacing, the deepest transmission minimum is reached at a strongly positive angle (above $60^{\circ}$ ), while the lattice is almost transparent $(90 \%$ transmission or more) at sharply negative angles. The fact that the angle of maximum and minimum transmission is rather insensitive to the lattice pitch indicates that while dipole-dipole interactions in the lattice may change the resonance frequency, width, and strength, they do not strongly modify the angle for addressing the highest pseudochiral contrast. Comparing the resonance frequency shift in Fig. 5(b) with the value of the transmission on resonance $T_{\min }$ in Fig. 5(a), it is seen that for angles close to the point where the lattice is almost transparent, the dipole-dipole coupling-induced frequency shift vanishes. This realization is consistent with the fact that at incident fields near transparency, hardly any dipole moment is set up. Conversely, we note that for any given lattice pitch, the maximum frequency shift is located at incidence normal to the lattice and not at the angle where the transmission minimum is deepest. This conclusion is not easily explained in a simple dipole hybridization model, ${ }^{51}$ since the frequency shift is a complex interplay of partially canceling transverse and longitudinal electric-dipole coupling (along $\hat{\boldsymbol{x}}$ and $\hat{\boldsymbol{y}}$, respectively), a weaker transverse magnetic dipole coupling (along $\hat{z}$ ), as well as magnetoelectric coupling that depends on the relative phase with which $\boldsymbol{p}$ and $\boldsymbol{m}$ are driven.

In order to compare the full lattice calculation with those of a single SRR, we calculated the extinction cross section as a function of input incident angle for six lattice spacings 


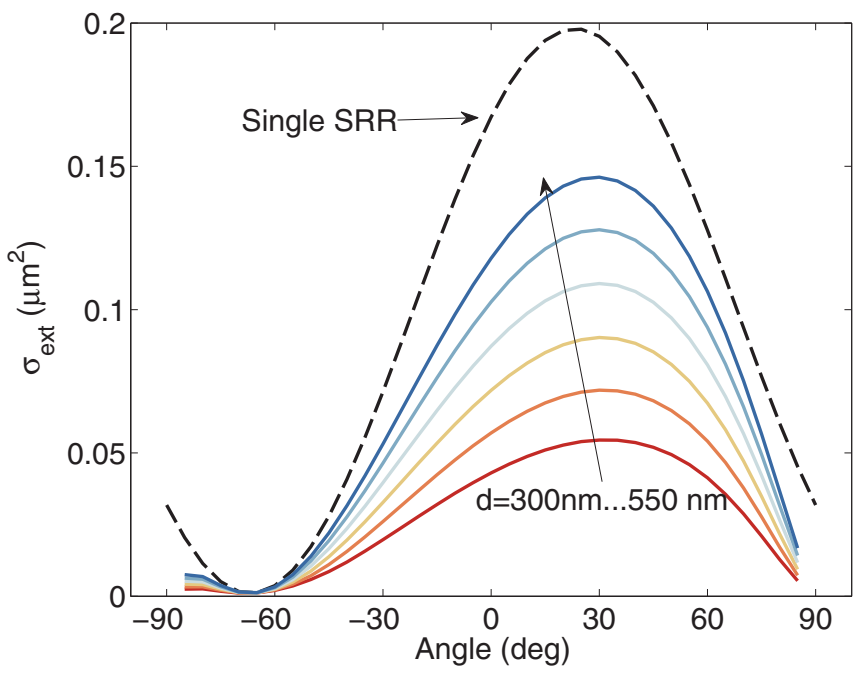

FIG. 6. (Color online) Calculated extinction cross section of a square lattice as a function of incident angle and lattice pitch for various lattice spacings $d$. Rotation is around the the symmetry axis of the SRR. Dashed and solid lines are for the single SRR and full lattice, respectively.

for the two scenarios. For the single SRR, we calculated the extinction cross section from the work done by the incident field, $W=2 \pi k\left\{\operatorname{Re}\left(\boldsymbol{E}_{\text {in }}, \boldsymbol{H}_{\text {in }}\right) \cdot \operatorname{Im}\left[\left(\boldsymbol{\alpha}\left(\boldsymbol{E}_{\text {in }}, \boldsymbol{H}_{\text {in }}\right)^{\top}\right]-\right.\right.$ $\left.\operatorname{Im}\left(\boldsymbol{E}_{\text {in }}, \boldsymbol{H}_{\text {in }}\right) \cdot \operatorname{Re}\left[\boldsymbol{\alpha}\left(\boldsymbol{E}_{\text {in }}, \boldsymbol{H}_{\text {in }}\right)^{\top}\right]\right\} / Z$, divided with the input intensity, $\left|\boldsymbol{E}_{\text {in }}\right|^{2} /(2 Z)$, where $Z$ is the impedance of the surrounding material. For the lattice calculations, we used Eq. (18) for the effective extinction cross section per SRR. We note that the factor $\cos \theta$ in this definition needs to be included to account for the simple geometrical projection argument that at larger angle, an incident beam of the same diameter intersects a larger set of split rings. The extracted effective extinction per split ring is presented in Fig. 6 for the case of rotation around the symmetry axis of the SRR. For the single SRR, the angular dependence on the extinction cross section can be characterized by a cosine shifted by roughly $20^{\circ}$ from the sample normal. ${ }^{41}$ This angle is much smaller than the angle away from the sample normal at which the maximum and minimum transmission is reached. This is only an apparent contradiction, since the trivial $\cos \theta$ projection effect causes a substantial additional skewing of the angular asymmetry in transmission in comparison to the asymmetry in per-building-block extinction. Indeed, the effective extinction cross section per split ring, corrected for the $\cos \theta$ projection factor, closely resembles the single SRR angle-dependent extinction, apart from being increasingly suppressed in amplitude for decreasing lattice pitch. This suppression of the peak extinction with increasing density is a consequence of super-radiant damping exactly as also evident for the normal-incidence data in Fig. 3(c). Even for the largest lattice spacings, there remains a significant difference between the extinction cross section of a single SRR and a SRR lattice, pointing to the importance of renormalization of the split ring response by retarded coherent interactions in full lattice calculations even when considering a dilute 2D metamaterial.

\section{CONCLUSION}

We have presented calculations of the full electromagnetic response of an infinite $2 \mathrm{D}$ magnetoelectric dipole lattice with consistent treatment of radiation damping, retardation, and energy conservation. The model was compared with recently published transmission data of split ring resonator (SRR) lattices with different lattice spacings. The model accounts excellently for the density-dependent collective resonance frequency, spectral width, and effective extinction cross section per split ring, in addition to capturing the strong pseudochiral response that fingerprints bianisotropic cross coupling. The model that we presented can be easily extended to deal with diffractive effects that occur at larger pitch, the emergence of surface lattice resonances, ${ }^{52,53}$ and to stacks of lattices or metasurfaces with more than one element per unit cell. In particular, we anticipate that the model is a semianalytical tool to explore the emergence, spectral and spatial dispersion of $\boldsymbol{\epsilon}$ and $\boldsymbol{\mu}$, and their dependence on the density and thickness of 3D metamaterials in a fully self-consistent electrodynamic multiple-scattering approach.

\section{ACKNOWLEDGMENTS}

This work is part of the research program of the Stichting voor Fundamenteel Onderzoek der Materie (FOM), which is financially supported by the Nederlandse Organisatie voor Wetenschappelijk Onderzoek (NWO). A.F.K. gratefully acknowledges a NWO-VIDI grant for financial support. P.L. acknowledges support by the Carlsberg Foundation as well as the Danish Research Council for Independent Research (Grant No. FTP 11-116740).

\section{APPENDIX A: SUMS OF MAGNETOELECTRIC DYADIC GREEN FUNCTION}

The sum presented in Eq. (8) requires special attention since it converges poorly. The problem has been treated extensively in Ref. 38 and utilizes a technique pioneered by Ewald. The technique consists in splitting a poorly convergent sum into two convergent terms, $\overleftrightarrow{\mathcal{G}}^{(1)}$ and $\overleftrightarrow{\mathcal{G}}^{(2)}$, which are exponentially convergent. Specifically, consider the sum

$$
\Gamma\left(\boldsymbol{k}_{\|}, \boldsymbol{r}\right)=\sum_{m, n} G^{0}\left(\boldsymbol{R}_{m n}-\boldsymbol{r}\right) e^{i \boldsymbol{k}_{\|} \cdot \boldsymbol{R}_{m n}},
$$

where the scalar Green function is

$$
G^{0}\left(\boldsymbol{R}_{m n}-\boldsymbol{r}\right)=\frac{e^{i k\left|\boldsymbol{R}_{m n}-\boldsymbol{r}\right|}}{\left|\boldsymbol{R}_{m n}-\boldsymbol{r}\right|} .
$$

We may rewrite this as

$$
\sum_{m, n} \frac{e^{i k\left|\boldsymbol{R}_{m n}-\boldsymbol{r}\right|}}{\left|\boldsymbol{R}_{m n}-\boldsymbol{r}\right|} e^{i \boldsymbol{k}_{\| \mid} \cdot \boldsymbol{R}_{m n}}=\Gamma^{(1)}+\Gamma^{(2)} .
$$

Here,

$$
\begin{aligned}
\Gamma^{(1)}= & \frac{\pi}{\mathcal{A}} \sum_{\tilde{m} \tilde{n}}\left\{\frac { e ^ { i ( \boldsymbol { k } _ { \| } + g _ { \tilde { m } \tilde { n } } ) \cdot \boldsymbol { r } _ { \| } } } { k _ { \tilde { m } \tilde { n } } ^ { z } } \cdot \left[e^{i k_{\tilde{m} \tilde{n}}^{z}|z|} \operatorname{erfc}\left(\frac{k_{\tilde{m} \tilde{n}}^{z}}{2 \eta}+|z| \eta\right)\right.\right. \\
& \left.\left.+e^{-i k_{\tilde{m} \tilde{n}}^{z}|z|} \operatorname{erfc}\left(\frac{k_{\tilde{m} \tilde{n}}^{z}}{2 \eta}-|z| \eta\right)\right]\right\}
\end{aligned}
$$


and

$$
\begin{aligned}
\Gamma^{(2)}= & \sum_{m n}\left\{\frac { e ^ { i \boldsymbol { k } _ { \| } \cdot \boldsymbol { R } _ { m n } } } { 2 \rho _ { m n } } \cdot \left[e^{i k \rho_{m n}} \operatorname{erfc}\left(\rho_{m n} \eta+\frac{i k}{2 \eta}\right)\right.\right. \\
& \left.\left.+e^{-i k \rho_{m n}} \operatorname{erfc}\left(\rho_{m n} \eta-\frac{i k}{2 \eta}\right)\right]\right\},
\end{aligned}
$$

where we used $\quad \boldsymbol{r}=\left(\boldsymbol{r}_{\|}, z\right), \quad k=\omega / c, \quad k_{\tilde{m} \tilde{n}}^{z}=$ $\sqrt{k^{2}-\left|\boldsymbol{k}_{\|}+\overleftrightarrow{g}_{\tilde{m} \tilde{n}}\right|^{2}}$, and $\rho_{m n}=\left|\boldsymbol{R}_{m n}-\boldsymbol{r}_{\|}\right|$. The convergence of Eqs. (A4b) and (A4a) follows from the asymptotic expansion of the error function revealing $z \operatorname{erfc}(z) \sim \exp \left(-z^{2}\right)$ for $z \rightarrow \infty .^{38}$ The parameter $\eta$ can be chosen for optimal convergence and should be set around $\eta=\sqrt{\pi} / a$, where $a$ is the lattice constant. Naturally, the cutoff for the summation over $m$ and $n$ must be chosen at least bigger than the number of propagating grating diffraction orders which one expects. For our calculations on metamaterials, with essentially no grating orders, i.e., $k a \leqslant 2 \pi$, we already obtained converged lattice sums for $|m, n| \leqslant 5$.

The dyadic lattice sums in Eq. (8) are easily generated by noting that the scalar Green function,

$$
G\left(\boldsymbol{r}, \boldsymbol{r}^{\prime}\right)=\frac{\exp \left(i k\left|\boldsymbol{r}-\boldsymbol{r}^{\prime}\right|\right)}{\left|\boldsymbol{r}-\boldsymbol{r}^{\prime}\right|},
$$

sets the dyadic Green function via

$$
\overleftrightarrow{G}^{0}\left(\boldsymbol{r}-\boldsymbol{r}^{\prime}\right)=\left(\begin{array}{cc}
\mathbb{I} k^{2}+\nabla \otimes \nabla & -i k \nabla \times \\
i k \nabla \times & \mathbb{I} k^{2}+\nabla \otimes \nabla
\end{array}\right) G\left(\boldsymbol{r}, \boldsymbol{r}^{\prime}\right)
$$

where $\mathbb{I}$ indicates the $3 \times 3$ identity matrix and $\otimes$ denotes the outer product. The derivatives can be simply pulled into each exponentially convergent sum to be applied to each term separately, and are most easily implemented in practice by noting that the sum $\Gamma^{(2)}$ only depends on radius in spherical coordinates $\rho_{m n}$, while the sum in $\Gamma^{(1)}$ only depends on radius and height in cylindrical coordinates. For these coordinate systems, the differential operator in Eq. (A6) take particularly simple forms. For spherical coordinates, this form reads

$$
\begin{aligned}
\left(\mathbb{I} k^{2}+\nabla \nabla\right) F(r)= & \mathbb{I}\left[k^{2} F(r)+\frac{1}{r} \frac{d}{d r} F(r)\right] \\
& +\left(\begin{array}{ccc}
x^{2} & x y & x z \\
x y & y^{2} & y z \\
x z & y z & z^{2}
\end{array}\right) \frac{1}{r} \frac{d}{d r}\left[\frac{1}{r} \frac{d}{d r} F(r)\right]
\end{aligned}
$$

and

$$
-i k \nabla \times F(r)=i k\left(\begin{array}{ccc}
0 & z & -y \\
-z & 0 & x \\
y & -x & 0
\end{array}\right) \frac{1}{r} \frac{d}{d r} F(r),
$$

which can be directly applied to the summands in Eq. (A4b). For cylindrical coordinates, the differential form reads

$$
\begin{aligned}
&\left(\mathbb{I} k^{2}+\nabla \otimes \nabla\right) e^{i \boldsymbol{k} \cdot \rho} g(z) \\
&=\left(\begin{array}{ccc}
k^{2}-k_{x}^{2} & -k_{x} k_{y} & 0 \\
-k_{x} k_{y} & k^{2}-k_{y}^{2} & 0 \\
0 & 0 & k^{2}
\end{array}\right) e^{i \boldsymbol{k} \cdot \boldsymbol{r}_{\|}} g(z) \\
&+\left(\begin{array}{ccc}
0 & 0 & i k_{x} \\
0 & 0 & i k_{y} \\
i k_{x} & i k_{y} & 0
\end{array}\right) e^{i \boldsymbol{k} \cdot \boldsymbol{r}_{\|}} \frac{d g(z)}{d z} \\
&+\left(\begin{array}{ccc}
0 & 0 & 0 \\
0 & 0 & 0 \\
0 & 0 & 1
\end{array}\right) e^{i \boldsymbol{k} \cdot \boldsymbol{r}_{\|}} \frac{d^{2} g(z)}{d z^{2}}
\end{aligned}
$$

and

$$
\begin{aligned}
-i k \nabla \times e^{i k \cdot \boldsymbol{r}_{\|}} g(z)= & \left(\begin{array}{ccc}
0 & 0 & -k k_{y} \\
0 & 0 & k k_{x} \\
k k_{y} & -k k_{x} & 0
\end{array}\right) e^{i k \cdot \boldsymbol{r}_{\|}} g(z) \\
& +\left(\begin{array}{ccc}
0 & i k & 0 \\
-i k & 0 & 0 \\
0 & 0 & 0
\end{array}\right) e^{i k r_{\|}} \frac{d g(z)}{d z}, \quad
\end{aligned}
$$

which can be directly applied to evaluate the dyadic equivalent of Eq. (A4a).

\section{APPENDIX B: FAR FIELD}

Carrying out the differentiation in Eq. (A6) and keeping only terms with $\left(\left|\boldsymbol{r}-\boldsymbol{r}^{\prime}\right| k\right)^{-1}$, we get

$$
\overleftrightarrow{G}_{\infty}^{0}\left(\boldsymbol{r}-\boldsymbol{R}_{m n}\right)=\overleftrightarrow{M}_{m n}^{(\infty)} G\left(\boldsymbol{r}, \boldsymbol{R}_{m n}\right),
$$

where [with $\boldsymbol{\xi}=\left(\boldsymbol{r}-\boldsymbol{R}_{m n}\right) /\left|\boldsymbol{r}-\boldsymbol{R}_{m n}\right|$ ]

$$
\overleftrightarrow{M}_{m n}^{(\infty)}=\left(\begin{array}{cc}
\overleftrightarrow{A} & \stackrel{\leftrightarrow}{B} \\
-\overleftrightarrow{B} & \stackrel{\leftrightarrow}{A}
\end{array}\right)
$$

with $\stackrel{\leftrightarrow}{A}=\mathbb{I}-\xi \otimes \xi, \quad$ and $\quad \stackrel{\leftrightarrow}{B}=\left(\begin{array}{ccc}0 & \xi_{z}-\xi_{y} & \\ -\xi_{z} & 0 & \xi_{x} \\ \xi_{y} & -\xi_{x} & 0\end{array}\right)$. *fkoenderink@amolf.nl

${ }^{1}$ V. G. Veselago, Sov. Phys. Usp. 10, 509 (1968).

${ }^{2}$ J. B. Pendry, Phys. Rev. Lett. 85, 3966 (2000).

${ }^{3}$ N. I. Zheludev and Y. S. Kivshar, Nat. Mater. 11, 917 (2012).

${ }^{4}$ J. B. Pendry, A. Aubry, D. R. Smith, and S. A. Maier, Science 337, 549 (2012).

${ }^{5}$ N. Liu, S. Kaiser, and H. Giessen, Adv. Mat. 20, 4521 (2008).
${ }^{6}$ N. Liu, H. Liu, S. Zhu, and H. Giessen, Nat. Photon. 3, 157 (2009).

${ }^{7}$ E. Plum, X.-X. Liu, V. A. Fedotov, Y. Chen, D. P. Tsai, and N. I. Zheludev, Phys. Rev. Lett. 102, 113902 (2009).

${ }^{8}$ N. Feth, M. König, M. Husnik, K. Stannigel, J. Niegemann, K. Busch, M. Wegener, and S. Linden, Opt. Express 18, 215 (2010).

${ }^{9}$ L. Langguth and H. Giessen, Opt. Express 19, 22156 (2011). 
${ }^{10}$ D. A. Powell, K. Hannam, I. V. Shadrivov, and Y. S. Kivshar, Phys. Rev. B 83, 235420 (2011).

${ }^{11}$ M. Decker, S. Linden, and M. Wegener, Opt. Lett. 34, 1579 (2009).

${ }^{12}$ M. Decker, N. Feth, C. M. Soukoulis, S. Linden, and M. Wegener, Phys. Rev. B 84, 085416 (2011).

${ }^{13}$ F. von Cube, S. Irsen, R. Diehl, J. Niegemann, K. Busch, and S. Linden, Nano Lett. 13, 703 (2013).

${ }^{14}$ A. V. Kildishev, A. Boltasseva, and V. M. Shalaev, Science 339, 1232009 (2013).

${ }^{15}$ N. Yu, F. Aieta, P. Genevet, M. A. Kats, Z. Gaburro, and F. Capasso, Nano Lett. 12, 6328 (2012).

${ }^{16}$ A. F. Koenderink, Nano Lett. 9, 4228 (2009).

${ }^{17}$ F. B. Arango, A. Kwadrin, and A. F. Koenderink, ACS Nano 6, 10156 (2012).

${ }^{18}$ S. A. Maier, M. D. Friedman, P. E. Barclay, and O. Painter, Appl. Phys. Lett. 86, 071103 (2005).

${ }^{19}$ N. Yu, P. Genevet, M. A. Kats, F. Aieta, J.-P. Tetienne, F. Capasso, and Z. Gaburro, Science 334, 333 (2011).

${ }^{20}$ F. Aieta, P. Genevet, M. A. Kats, N. Yu, R. Blanchard, Z. Gaburro, and F. Capasso, Nano Lett. 12, 4932 (2012).

${ }^{21}$ J. K. Gansel, M. Thiel, M. S. Rill, M. Decker, K. Bade, V. Saile, G. von Freymann, S. Linden, and M. Wegener, Science 325, 1513 (2009).

${ }^{22}$ M. A. Kats, P. Genevet, G. Aoust, N. Yu, R. Blanchard, F. Aieta, Z. Gaburro, and F. Capasso, Proc. Natl. Acad. Sci. USA 109, 12364 (2012).

${ }^{23}$ M. Schäferling, D. Dregely, M. Hentschel, and H. Giessen, Phys. Rev. X 2, 031010 (2012).

${ }^{24}$ E. Plum, V. A. Fedotov, and N. I. Zheludev, J. Opt. 13, 024006 (2011).

${ }^{25}$ B. Lahiri, S. G. McMeekin, A. Z. Khokhar, R. M. De La Rue, and N. P. Johnson, Opt. Express 18, 3210 (2010).

${ }^{26}$ M. W. Klein, C. Enkrich, M. Wegener, C. M. Soukoulis, and S. Linden, Opt. Lett. 31, 1259 (2006).

${ }^{27}$ S. Linden, C. Enkrich, M. Wegener, J. Zhou, T. Koschny, and C. M. Soukoulis, Science 306, 1351 (2004).

${ }^{28}$ C. Enkrich, M. Wegener, S. Linden, S. Burger, L. Zschiedrich, F. Schmidt, J. F. Zhou, T. Koschny, and C. M. Soukoulis, Phys. Rev. Lett. 95, 203901 (2005).

${ }^{29}$ I. Sersic, M. Frimmer, E. Verhagen, and A. F. Koenderink, Phys. Rev. Lett. 103, 213902 (2009).

${ }^{30} \mathrm{~F}$. Capolino, Theory and Phenomena of Metamaterials, edited by F. Capolino (CRC, Boca Raton, FL, 2009).

${ }^{31}$ R. Zhao, T. Koschny, and C. M. Soukoulis, Opt. Express 18, 1513 (2010).
${ }^{32}$ D. R. Smith, D. C. Vier, T. Koschny, and C. M. Soukoulis, Phys. Rev. E 71, 036617 (2005).

${ }^{33}$ C. Rockstuhl, T. Zentgraf, H. Guo, N. Liu, C. Etrich, I. Loa, K. Syassen, J. Kuhl, F. Lederer, and H. Giessen, Appl. Phys. B 84, 219 (2006).

${ }^{34}$ M. Husnik, M. W. Klein, N. Feth, M. König, J. Niegemann, K. Busch, S. Linden, and M. Wegener, Nat. Photon. 2, 614 (2008).

${ }^{35}$ L. Novotny and B. Hecht, Principles of Nano-Optics (Cambridge University Press, New York, 2008).

${ }^{36}$ I. Sersic, C. Tuambilangana, T. Kampfrath, and A. F. Koenderink, Phys. Rev. B 83, 245102 (2011).

${ }^{37}$ A. F. Koenderink and A. Polman, Phys. Rev. B 74, 033402 (2006).

${ }^{38}$ C. M. Linton, SIAM Rev. 52, 630 (2010).

${ }^{39}$ B. Gompf, J. Braun, T. Weiss, H. Giessen, M. Dressel, and U. Hübner, Phys. Rev. Lett. 106, 185501 (2011).

${ }^{40}$ F. J. García de Abajo, Rev. Mod. Phys. 79, 1267 (2007).

${ }^{41}$ I. Sersic, M. A. van de Haar, F. B. Arango, and A. F. Koenderink, Phys. Rev. Lett. 108, 223903 (2012).

${ }^{42}$ A. Serdyukov, I. Semchenko, S. Tretyakov, and A. Sihvola, Electromagnetics of Bi-anisotropic Materials: Theory and Applications, 1st ed., edited by D. de Cogan, Electrocomponent Science Monographs (Gordon and Breach, Amsterdam, 2001).

${ }^{43}$ R. Marqués, F. Medina, and R. Rafii-El-Idrissi, Phys. Rev. B 65, 144440 (2002).

${ }^{44}$ J. E. Sipe and J. V. Kranendonk, Phys. Rev. A 9, 1806 (1974).

${ }^{45}$ P. Belov, S. Maslovski, K. Simovski, and S. Tretyakov, Tech. Phys. Lett. 29, 718 (2003).

${ }^{46}$ F. B. Arango and A. Femius Koenderink, New J. Phys. 15, 073023 (2013).

${ }^{47}$ S. Y. Park and D. Stroud, Phys. Rev. B 69, 125418 (2004).

${ }^{48}$ N. Katsarakis, T. Koschny, M. Kafesaki, E. N. Economou, and C. M. Soukoulis, Appl. Phys. Lett. 84, 2943 (2004).

${ }^{49}$ T. Verbiest, M. Kauranen, Y. Van Rompaey, and A. Persoons, Phys. Rev. Lett. 77, 1456 (1996).

${ }^{50}$ S. Tretyakov, A. Sihvola, A. Sochava, and C. Simovski, J. Electromagnet. Wave 12, 481 (1998).

${ }^{51}$ E. Prodan, C. Radloff, N. J. Halas, and P. Nordlander, Science 302, 419 (2003).

${ }^{52}$ S. R. K. Rodriguez, A. Abass, B. Maes, O. T. A. Janssen, G. Vecchi, and J. Gómez Rivas, Phys. Rev. X 1, 021019 (2011).

${ }^{53}$ G. Lozano, D. J. Louwers, S. R. Rodríguez, S. Murai, O. T. Jansen, M. A. Verschuuren, and J. Gómez Rivas, Light: Sci. Appl. 2, e66 (2013). 